\title{
Development of X-TOOLSS: Preliminary Design of Space Systems Using Evolutionary Computation
}

\author{
Andrew R. Schnell ${ }^{1}$, Patrick V. Hull ${ }^{2}$, and Mike L. Tinker ${ }^{3}$ \\ Marshall Space Flight Center, Huntsville, AL 35812 \\ Gerry Dozier ${ }^{4}$ \\ North Carolina Agricultural and Technical State University, Greensboro, NC 27411 \\ Lauren Alverson ${ }^{5}$ \\ Auburn University, Auburn, AL 36849 . \\ Aaron Garrett ${ }^{6}$ \\ Jacksonville State University, Jacksonville, AL 36265 \\ and \\ Jarred Reneau ${ }^{7}$ \\ Mississippi State University, Mississippi State, MS 39762
}

Evolutionary computational (EC) techniques such as genetic algorithms (GA) have been identified as promising methods to explore the design space of mechanical and electrical systems at the earliest stages of design. In this paper the authors summarize their research in the use of evolutionary computation to develop preliminary designs for various space systems. An evolutionary computational solver developed over the course of the research, X-TOOLSS (Exploration Toolset for the Optimization of Launch and Space Systems) is discussed. With the success of early, low-fidelity example problems, an outline of work involving more computationally complex models is discussed.

\section{Nomenclature}

$\begin{array}{lll}C S & = & \text { Candidate Solution } \\ \text { DAKOTA } & = & \text { Design Analysis Kit for Optimization and Terascale Applications } \\ \text { EC } & = & \text { Evolutionary Computation } \\ \text { GEC } & = & \text { Genetic \& Evolutionary Computations } \\ \text { NEP } & = & \text { Nuclear Electric Propulsion } \\ \text { USSEP } & = & \text { U.S. Space Exploration Policy } \\ \text { VSE } & = & \text { Vision for Space Exploration } \\ X-T O O L S S & = & \text { eXploratory Toolset for the Optimization Of Launch and Space Systems }\end{array}$

\section{Introduction}

$\triangle$ CCORDING to Dozier, et al., genetic and evolutionary computation (GEC) is "the field of study

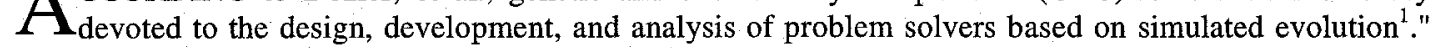
Some of the cited benefits of evolutionary computation are: the ability to handle discontinuous problems, problems with multiple local minima, and problems involving discrete variables. ${ }^{2}$ The use of GECs to expedite the design of complex launch and space systems is being investigated.

Unlike traditional optimization techniques, GECs work with a population of candidate solutions (CS). Initially, a population of CSs are generated and rated against a user-defined evaluation function. The

\footnotetext{
${ }^{1}$ Universities Space Research Association, ES22, MSFC, Huntsville, AL 35812, AIAA Member

${ }^{2}$ EV32, Marshall Space Flight Center, Huntsville, AL 35812, AIAA Member

${ }^{3}$ Branch Chief, ES22, Marshall Space Flight Center, Huntsville, AL 35812, AIAA Associate Fellow

${ }^{4}$ Professor, Department of Computer Science, NCATSU, Greensboro, NC 27411

${ }^{5}$ Student, Department of Electrical and Computer Engineering, Auburn University, Auburn, AL 36849

${ }^{6}$ Assistant Professor, Department of Mathematics, Computing, and Information Sciences, Jacksonville State, AL 36265

${ }^{7}$ Student, Mississippi State University, AIAA Student Member
} 
candidates' "fitness" is then ranked. Candidates with a high fitness are designated parent designs, which are allowed to make offspring designs based on crossover or mutation. The offspring are then evaluated and assigned a fitness and replace weaker members of the population. This process continues until a userspecified stopping criteria has been met.

To consider the appropriateness of the use of evolutionary computing on the multi-disciplinary design of space hardware, a series of projects has been developed that test the deployment of evolutionary computing techniques. These projects are discussed in Section 2. The first project in Section 2A involves developing code that solves benchmark optimization problems. Then, two multidisciplinary projects involving the use of EC to search the design space are discussed. In Section 2B, the design of a nuclear electric propulsion spacecraft is discussed. In Section $2 \mathrm{C}$, the design of a lunar habitat is presented.

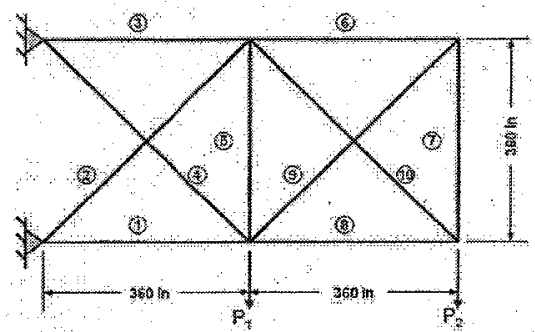

(a)

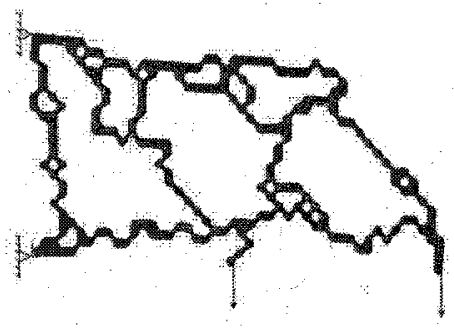

(b)

Figure 1. (a) 10-bar truss mass minimization problem. (b) Alternative topological solution for the $\mathbf{1 0}$ bar problem.

In Section 3, we discuss a computational solver, X-TOOLSS, developed over the course of this research. X-TOOLSS (eXploration Toolkit for the Optimization of Launch and Space Systems) is a Javabased computer program employing twelve evolutionary computational algorithms.

In Section 4, we describe future work. Future work will continue to be focused on the sizing of a future lunar habitat. More complex computational models that better reflect the characteristics of the design will be developed.

\section{Projects}

In this section, several projects undertaken by the mechanical and thermal analysts at MSFC to study the usefulness of genetic algorithms in the exploration of design alternatives are summarized. Example problems range in complexity from simple two-variable mathematical optimization problems to multiplatform multi-objective analyses.

Table 1. Comparison of solutions to 10-bar truss problem.

\begin{tabular}{|c|c|c|c|c|c|c|}
\hline \multirow{2}{*}{$\begin{array}{c}\text { Bar } \\
\text { number }\end{array}$} & \multicolumn{6}{|c|}{ Optimum cross section areas of bars, inch ${ }^{2}$} \\
\hline & $\begin{array}{c}\text { Schmit, } \\
\text { Miura }\end{array}$ & $\begin{array}{l}\text { Schmit, } \\
\text { Farchi }\end{array}$ & Venkayya & Haug, Arora & $\begin{array}{l}\text { Software } \\
\text { SOOOPT }\end{array}$ & 8.8 \\
\hline 1 & 24.43 & 24.25 & 23.4 & 23.27 & 23.93 & 21.6 .59 \\
\hline 2 & 21.06 & 20.69 & 21.08 & 21.2 & 20.96 & 28,3128 \\
\hline 3 & 30.66 & 33.42 & 30.41 & 30.03 & 30.74 & 21.6214 \\
\hline 4 & 8.58 & 8.39 & 8.69 & 7.47 & 8.53 & 24.0098 \\
\hline 5 & 0.1 & 0.1 & 0.1 & 0.1 & 0.1 & 0.3671 \\
\hline 6 & 0.1 & 0.1 & 0.13 & 0.1 & 0.1 & 0.1 \\
\hline 7 & 0.1 & 0.1 & 0.1 & 0.56 & 0.1 & 011 \\
\hline 8 & 14.59 & 14.26 & 14.9 & 15.29 & 14.74 & 15.798 \\
\hline 9 & 0.1 & 0.1 & 0.19 & 0.1 & 0.1 & 0.1 \\
\hline 10 & 21.06 & 20.69 & 21.08 & 21.2 & 20.96 & $24(0) 1$ \\
\hline $\begin{array}{c}\text { Weight, } \\
10^{3} \\
\text { pound }\end{array}$ & 5.074 & 5.092 & 5.088 & 5.061 & 5.074 & 5.83 \\
\hline
\end{tabular}

\section{A. Refined Truss Design}

To build confidence in the use of evolutionary computation, a series of increasingly complex benchmark mathematical optimization problems are solved using $\mathrm{X}$-TOOLSS, and their results compared to other solutions. A ten-bar benchmark mass minimization truss problem was selected and solved due to its prevalence in the literature. ${ }^{3,4,5,6,7} \quad \mathrm{~A}$ representation of the problem is shown in Figure 1. The designer seeks a truss

that meets the given loading condition while minimizing the overall weight of the truss. When a GA technique was used to solve the 10-bar truss problem, its results were close enough to published results to provide confidence in the method. ${ }^{8}$ Further refinement of the problem ${ }^{9}$ results in a novel solution shown in Figure 2. A comparison of the results compared to the solutions in the literature is shown in Table 1. 


\section{B. NEVOT}

The first multi-disciplinary deployment of $\mathrm{EC}$ in this research was in the development of a design for a nuclear electric propulsion (NEP) spacecraft ${ }^{\mathbf{1 0 1 1 , 1 2}}$. The conceptual layout of an NEP vehicle is shown in Figure 2. This design exploration involved seeking a design with a minimum amount of mass while seeking a maximum amount of thrust, and appropriate values for the thickness of reactor shield, the length of the radiator, and the dimensions of the habitat module.

NEVOT was a joint effort of Marshall Space Flight Center, Oak Ridge National Laboratories, and the Arnold Engineering Development Center. There were seven main analysis modules: Trajectory, Space Reactor Power System (SRPS), Power Management and Distribution (PMAD), Electric Propulsion (EP), Habitat (HAB), Truss (TRUSS), and Configuration (CONFIG). A simulation executive controlled input and output files. The genetic algorithm solver used for the optimization was Sandia Laboratories' Design Analysis Kit for Optimization and Terascale Applications (DAKOTA) software package. A

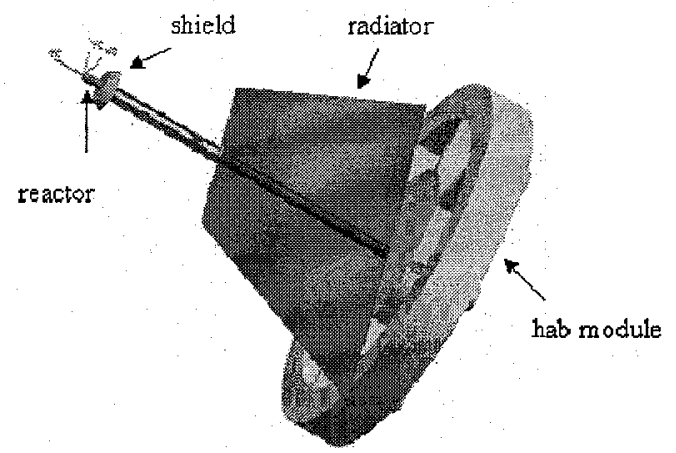

Figure 2. Typical nuclear electric propulsion vehicle layout. schematic of the DAKOTA package with the NEVOT modules is shown in Figure 3. The multiple subsystem analysis codes were kept as modular as possible, facilitating modifications to the code when better data became available. Initial results were preliminary due to the limited fidelity of the subsystem modules; however, the usefulness of the approach in the context of preliminary design was shown.

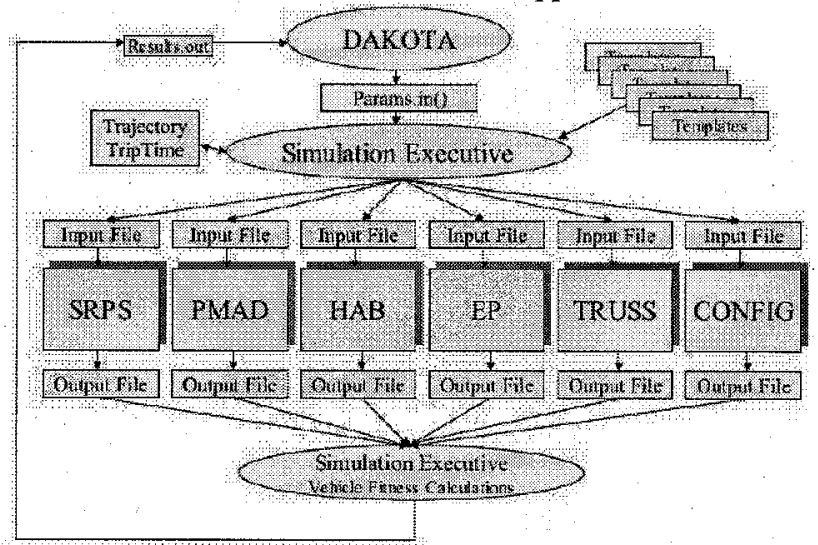

Figure 3. Schematic representing relationships of the components of NEVOT.
The methodology used for developing NEVOT allowed investigation of subsystems not typically coded together. Experimenting with truly unique vehicle combinations allows designers to find new and better solutions.

Results of the NEVOT work were considered preliminary due to the coarse nature of the sub-module programs developed.

\section{Lunar Habitat}

The most current motivating example for this research is the design of a lunar habitat. The example was chosen in light of the then recent announcement of the "Vision for Space Exploration" (VSE, now known as the United States Space Exploration Policy USSEP) which calls for a human return to the Moon by 2020 and a permanent lunar base. Over the course of USSEP, the length of lunar surface missions would extend from days to several months. An important impediment to overcome in longer duration missions would be the threat posed by the harsh environment found on the Moon. The use of available lunar materials, namely the lunar regolith, is a priority due to the fact that the mass of materials needed in construction would be too great to be feasibly transported from Earth. 


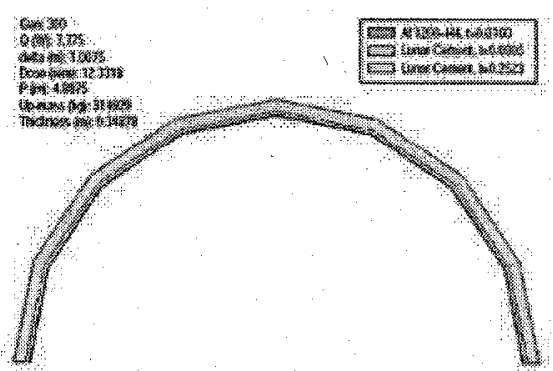

Figure 4. Example design of lunar habitat.

In this preliminary design exercise, a sample design is shown in Figure 4, a dome-shaped structure is deployed and then buried under lunar regolith. In this scenario, designers seek configurations and dimensions of proposed lunar habitat construction materials that minimize thermal losses, have good structural integrity, and adequate protection from meteoroid impact and radiation. As in the NEVOT example, subsystem analysis codes were developed to estimate each design's fitness for each of the above requirements. The overall objective was to minimize each design's up-mass, the mass of the components to be launched from Earth. The computational architecture utilized for this work is shown in Figure 5. Again, results were preliminary due to the low fidelity of the sample models. Results tended to side towards the heavy use of lunar regolith, which was expected due to the large emphasis on minimizing upmass.

Another unique aspect of this research was the generation of a meta-GA algorithm. The meta-GA algorithm allows the X-TOOLSS user to find values for the GA parameters that would provide faster results.

\section{X-TOOLSS}

$\mathrm{X}$-TOOLSS is a platform-independent evolutionary computational solver developed by the Applied Computational Intelligence Laboratory at Auburn University and developed over the course of this work. It is being further developed by the Department of Computer Science and the Applied Computational Intelligence Laboratory at North Carolina A\&T University in Greensboro, North Carolina.

In this section, X-TOOLSS and its capability are introduced. X-TOOLSS is a collection of twelve evolutionary computations in the form of a generational

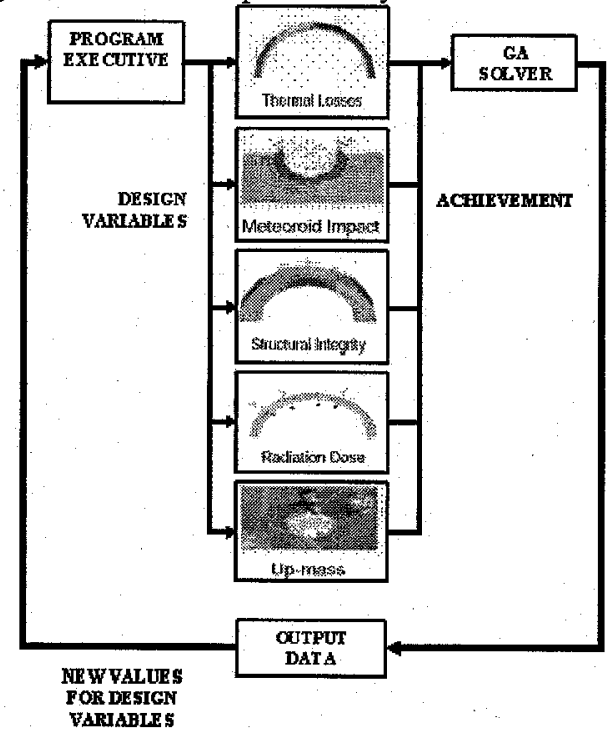

Figure 5. Computational architecture for lunar habitat example. GA, three steady-state GAs, a steady-generational GA, a particle swarm optimizer (PSO), two forms of differential evolutionary algorithms, an estimation of distribution algorithm (EDA), and four evolutionary

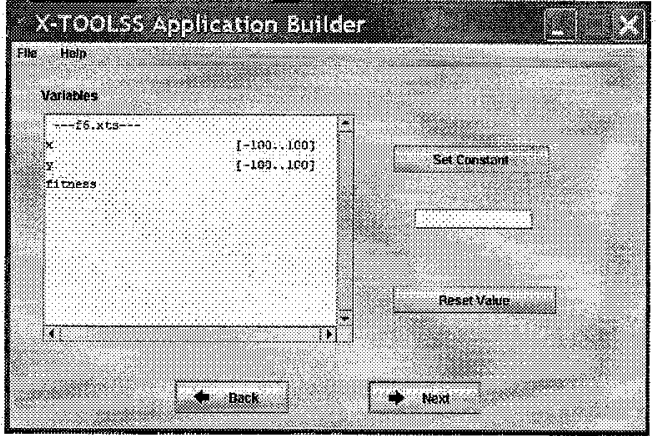

(a)

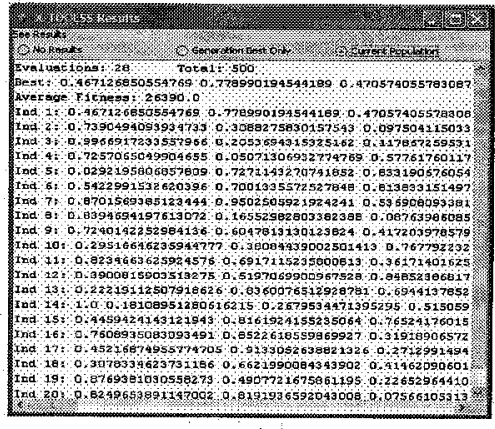

(b)

Figure 6 (a) User interface for X-TOOLSS (b) Sample results window for X-TOOLSS programming (EP) methods. Several variants of X-TOOLSS have been developed for solving a wide variety of problems. The X-TOOLSS graphical user interface (GUI) allows users to drag-and-drop problems specified in XTS files and then allows users to: (a) select a particular evolutionary computation for solving the problem and (b) specify to control parameters of the selected evolutionary computation 
(such as population size, crossover usage rate, mutation rate, etc.). X-TOOLSS also has a command-line interface which allows it to be executed from other software. X-TOOLSS also comes with the X-TOOLSS memespace utility that allows for the development of distributed/parallel evolutionary computations. The $\mathrm{X}$-TOOLSS memespace utility allows any number of ECs (running on the same or different computers) to communicate their best candidate solutions with one another. This results in distributed/parallel evolutionary computation and faster results. A sample GUI for X-TOOLSS is shown in Figure 6A, and the results window for a typical problem solved by X-TOOLSS is shown in Figure 6B.

\section{Future Work}

In this section some of the future work for this research is discussed. As mentioned in the previous section, previous examples have resulted in good proof-of-concept for the use of GEC in preliminary design; however, the results are deemed preliminary due to the low fidelity of subsystem models. The reasons for using low-fidelity models were twofold: the purpose of the previous research was to build confidence in the approach, and the long estimated computation time required for using higher fidelity models. Future example problems will extend the complexity of the models. Where currently twodimensional cross-section models are run, investigation of the addition of three-dimensional models will be accomplished An example of a three dimensional model of a lunar habitat is shown in Figure 7.

We will continue to compare the EC approach with other methods to explore the design space. We want to be able to easily analyze the trends that develop between design variables. To that end, we will also

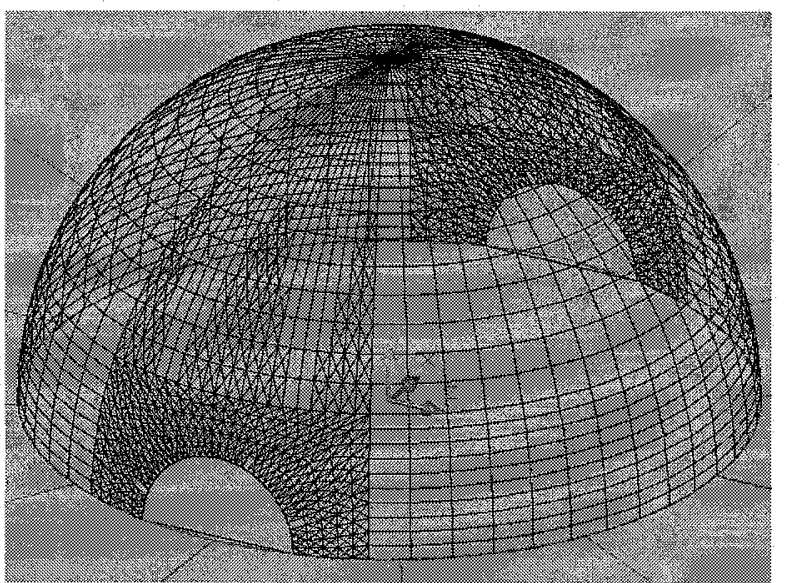

Figure 7. Sample three dimensional model of proposed lunar habitat for use in thermal radiation modeling. begin to explore new ways to visualize the results of the analyses.

More analysis modules will be added to the analysis. Considering life support requirements, shapes of the habitat, length of mission stays, the oxygen permeability of the material used on the habitat, and other characteristics of potential designs gives engineers more understanding of the candidates' performance. Improvement of the user interface with X-TOOLSS will continue, in order to reduce the lead time needed to encode the example problems.

An additional research avenue to be explored is the utilization of lunar reconnaissance data to build a methodology for selecting the physical location of the lunar habitat using GEC. Using data collected by reconnaissance spacecraft, it is planned to establish the local variations in the topology, chemical composition, and radiation environments at prospective sites, then use GEC to custom design candidate solutions for lunar habitats at these prospective sites.

\section{Acknowledgments}

The authors acknowledge the contributions of Andrew Benford, Ryan Irwin, Michael P. SanSoucie.

\section{References}

1. Dozier, G., Homaifar, A., Tunstel, E., and Battle, D. (2001). "Chapter 17: An Introduction to Evolutionary Computation," Intelligent Control Systems Using Soft Computing Methodologies, Zilouchian, A., and Jamshidi, M. (eds.) CRC Press, pp. 365-380.

2. Rowell, L.F., and Korte, J.J. (2003). "Launch Vehicle Design and Optimization Methods and Priority for the Advanced Engineering Environment." NASA/TM-2003-212654.

3. $\quad$ Schmit, L.A., and Miura, H. (1976). "A New Structural Analysis/Synthesis Capability --ACCESS 1." AIAA Journal. Vol. 14, No. 4. pp. 661-671.

4. Schmit, L.A., and Miura, H. (1976). "A New Structural Analysis/Synthesis Capability -- ACCESS 2." AIAA Journal. Vol. 14, No. 2. pp. 353-377. 
5. Schmit, L.A., and Farshi, B. (1974), "Some Approximation Concepts for Structural Synthesis." AIAA Journal. Vol. 12; pp. 692-699.

6. Venkayya, V.B. (1971). "Design of Optimum Structures." Computers and Structures. Vol. 1. pp. 265-309.

7. Arora, J.S. and Haug, Jr., E.J. (1976). "Efficient Optimal Design of Structures by Generalized Steepest Descent Programming." International Journal of Numerical Methods in Engineering. Vol. 10. pp. 747766.

8. Benford, A. and Tinker, M.L., (2004). "Truss Optimization for a Manned Nuclear Electric Space Vehicle Using Genetic Algorithms." 10th AIAA/ISSMO Multidisciplinary Analysis and Optimization Conference, Albany, NY. Paper No. AIAA 2004-4532.

9. Hull, P.V., Tinker, M.L., and Dozier, G.V. (2007). "Evolutionary Optimization of a Geometrically Refined Truss." NASA/TP-2007-214960.

10. Irwin, R.W., and Tinker, M.L. (2005). "Preliminary Design of a Manned Nuclear Electric Propulsion Vehicle Using Genetic Algorithms. Space Technology and Applications International Forum STAIF. Albuquerque, NM. American Institute of Physics Conference Proceedings. Vol. 746 pp. 983-990.

11. SanSoucie, M.P., Hull, P.V., Irwin, R.W., Tinker, M.L., and Patton, B.W., (2005), "Trade Studies for a Manned High-Power Nuclear Electric Propulsion Vehicle." 1st Space Exploration Conference: Continuing the Voyage of Discovery, AIAA, Orlando, FL. Paper No. AIAA 2005-2729.

12. Tinker, M.L., Steincamp, J.W., Stewart, E.T., Patton, B.W., Pannell, W.P., Newby, R.L., Coffman, M.E., and Kos, L.D., (2004), "Nuclear Electric Vehicle Optimization Toolset (NEVOT)." 10th AIAA/ISSMO Multidisciplinary Analysis and Optimization Conference, Albany, NY. Paper No. AIAA 2004-4552.

13. SanSoucie, M.P., Hull, P.V., Tinker, M.L., and Dozier, G.V., (2007), "Lunar Habitat Optimization Using Genetic Algorithms." NASA/TP-2007-214852.

14. Dozier, G., Bryant, K., Barksdale, J., Garrett, A., Tann, G., Brown, M., Meeks, R., Huang, S.-Y., Zou, X., Goff, L., Tinker, M., and Schnell, A., (2008). "AttackAids@A\&T, nk-HIV Docking, and X-TOOLSS," In Review: Midwest Artificial Intelligence and Cognitive Science Conference (MAICS-08). 